\title{
EchoGéo
}

\section{Les dynamiques urbaines de Shanghai : trois artères péri-centrales en mutation}

\section{Howard Vazquez}

\section{(2) OpenEdition}

1 Journals

Édition électronique

URL : https://journals.openedition.org/echogeo/11821

DOI : 10.4000/echogeo.11821

ISSN : 1963-1197

\section{Éditeur}

Pôle de recherche pour l'organisation et la diffusion de l'information géographique (CNRS UMR 8586)

Référence électronique

Howard Vazquez, "Les dynamiques urbaines de Shanghai : trois artères péri-centrales en mutation », EchoGéo [En ligne], 12 | 2010, mis en ligne le 31 mai 2010, consulté le 31 juillet 2021. URL : http:// journals.openedition.org/echogeo/11821; DOI : https://doi.org/10.4000/echogeo.11821

Ce document a été généré automatiquement le 31 juillet 2021.

EchoGéo est mis à disposition selon les termes de la licence Creative Commons Attribution - Pas d'Utilisation Commerciale - Pas de Modification 4.0 International (CC BY-NC-ND) 


\title{
Les dynamiques urbaines de Shanghai : trois artères péri- centrales en mutation
}

\author{
Howard Vazquez
}

\section{Introduction}

1 Shanghai, métropole en pleine mutation située dans un pays émergent, est le symbole $\mathrm{du}$ modernisme et du développement actuel chinois. Avec près de 14,5 millions $\mathrm{d}^{\prime}$ habitants ${ }^{1}$, Shanghai est aujourd'hui le symbole du dynamisme économique d'une Chine ouverte sur l'extérieur. Pôle structurant de la mondialisation et de la façade maritime chinoise, la ville connaît depuis 1992, date du voyage de Deng Xiaoping dans le Sud du pays, de nouvelles dynamiques urbaines. Vitrine de l'économie chinoise, Shanghai se caractérise par des transformations urbaines et sociales très rapides.

2 L'une des images qu'évoque la ville nous est livrée par la rue. L'évolution morphologique et fonctionnelle de la rue, en tant qu'espace public, suit celle de la société chinoise. Dès lors, les étapes de son histoire coïncident avec celles de l'histoire de la ville et de son urbanisation.

3 Au-delà de la simple verticalisation de l'espace bâti et des politiques de rénovation urbaine, les rues participent activement à la modernisation de Shanghai. Lieu privilégié pour aborder les mutations de la société chinoise, la rue est le propre de la ville, sa forme élémentaire, la plus simple unité en laquelle la forme urbaine puisse se résumer tout en nous livrant les mécanismes de son fonctionnement, voire nous révélant sa réalité essentielle ${ }^{2}$. Les acteurs publics et privés ont un impact prépondérant sur la morphologie des rues et l'organisation de l'espace.

4 Armatures de la ville, les artères péri-centrales symbolisent la modernisation intraurbaine de Shanghai et l'urbanité d'une certaine Chine. Les rues étudiées sont des artères péri-centrales, c'est-à-dire des axes importants, ouverts à l'espace public, menant au centre-ville de la métropole chinoise. Par leur multifonctionnalité, les 
artères péri-urbaines permettent de rendre compte des dynamiques urbaines. À l'heure de la mondialisation, cet article privilégie les artères péri-centrales aux rues piétonnes. En effet, les artères péri-centrales recouvrent plusieurs spécificités ainsi qu'une certaine conception de la riveraineté. Par riveraineté, on entend les interactions entre l'usage de la rue et celui de son environnement social et morphologique. Les singularités des artères péri-centrales recouvrent plusieurs aspects : la circulation et la gestion des flux, les usages et les offres de la rue, l'articulation entre le bâti et l'espace public que constitue la rue, et enfin la rue comme lieu de sociabilité. Ces rues, à la fois vivantes et quotidiennes, participent activement à la vie urbaine de la métropole et au processus de mondialisation. Dès lors, traiter l'évolution morphologique, fonctionnelle et sociale de trois artères péri-urbaines de Shanghai est révélateur des mutations observées à l'échelle de l'ensemble de la ville.

Une des spécificités de la mondialisation tient à sa tendance à l'uniformisation. Les dynamiques de la globalisation touchent notamment les rues. Même si chaque pays se réattribue de grandes enseignes mondialement connues ${ }^{3}$, en intégrant des marqueurs de standing international (Mc Donald's, Starbucks, KFC), les rues ont tendance à perdre leurs caractéristiques locales. L'objectif de cette démarche est d'analyser les transformations contemporaines des artères péri-centrales de Shanghai ${ }^{4}$.

Quelles sont les dynamiques à la fois sociales, politiques et économiques qui sont en jeu ? Quelles sont les fonctions, la nature et l'origine de ces espaces publics? En quoi les artères péri-centrales sont-elles représentatives de la ville? Comment les artères périurbaines de Shanghai ont-elles évolué depuis le début des années 1990 dans le cadre de la redéfinition plus globale de la municipalité ?

7 Afin de répondre à ces questions, nous allons nous concentrer, dans une première partie à l'approche théorique des artères péri-centrales de Shanghai. Dans une seconde partie, nous examinerons comment ces rues participent activement à la vie de la métropole chinoise. Dans une troisième partie, nous verrons dans quelle mesure ces rues sont des vecteurs de la mondialisation.

\section{Des rues en mouvement perpétuel}

\section{Des axes de circulation incontournables}

8 La rue comme tout espace public est avant tout un espace physique. Le terme espace public désigne les espaces de rassemblement ou de passage, ouverts à tous les usagers. $\mathrm{Au}$ sein d'une ville, les rues se caractérisent par une circulation et une gestion des flux de natures et d'intensités diverses. De même, par leur positionnement par rapport au centre-ville, les voies urbaines ont des largeurs et des liaisons différentes entre le centre et la périphérie. Les rues de Nankin (Ouest), Huaihai (Ouest) et celle du Sichuan (Nord), peuvent être qualifiées d'artères péri-centrales par rapport au centre historique de Shanghai. Elles n'appartiennent pas au centre-ville stricto sensu de la métropole chinoise. 
Carte 1 - Localisation des artères péri-centrales dans la ville-centre de Shanghai.

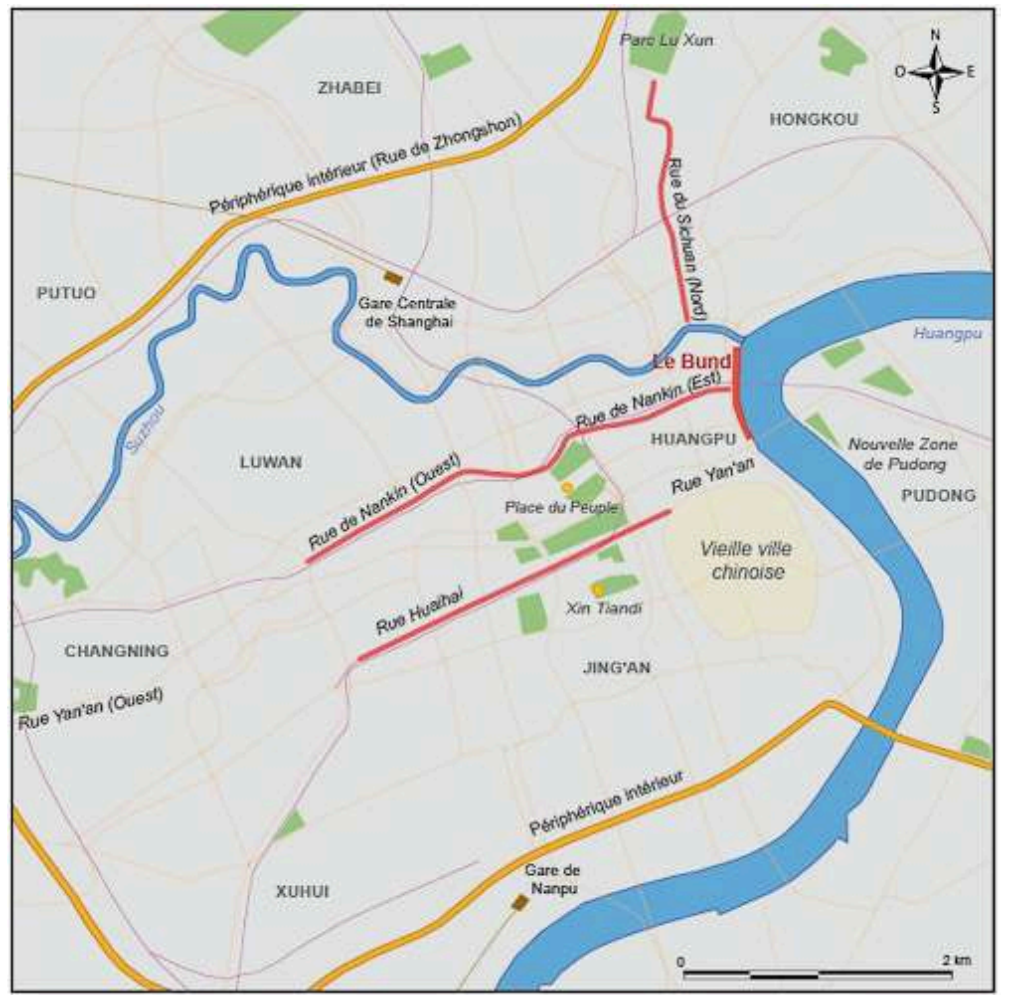

Du nord au sud : la rue du Sichuan (Nord), la rue de Nankin (Ouest), et la rue Huaihai (Ouest)

Réalisation : Howard Vazquez

9 Au niveau morphologique, ce sont des rues qui forment l'ossature principale du paysage urbain. En effet, ce sont des radiales qui rayonnent à partir du centre. Dès lors, les artères péri-centrales apparaissent comme des axes de circulation rapides et incontournables permettant de circuler du centre-ville vers la périphérie. Les artères péri-centrales de Shanghai sont irriguées par un réseau de rues et de ruelles, et s'orientent ensuite, à leur tour, vers les arrondissements périphériques. Ce sont des voies qui ont pour objet la communication entre les différentes zones de la ville. Elles s'organisent en axe, comme les branches d'un arbre, facilitant ainsi la circulation des flux.

L'analyse des artères péri-centrales de Shanghai nécessite de les réinscrire à l'échelle de la ville et du quartier dans lequel elles se situent:

- La rue de Nankin (Nanjinglu), longue de près de 8 kilomètres, se compose à la fois d'un secteur est (Nanjingdonglu) et d'un secteur ouest (Nanjinxilu). Le tronçon oriental qui s'étend de la place du Peuple (Renmin guangchang) jusqu'au Bund est en partie une rue piétonne à vocation touristique. Vitrine de Shanghai, la rue de Nankin (Est) est une rue monofonctionnelle. La partie occidentale, objet de cette démarche, s'étend de la place du Peuple jusqu'au quartier du temple Jing'an. Située dans l'ancienne concession internationale, la rue de Nankin (Ouest) est l'axe commercial le plus international de Shanghai. Il se compose de centres d'affaires (bureaux, administrations), de centres commerciaux ultramodernes (le CITIC Plaza, le Meilongzhen et le Plaza 66) et de boutiques de luxe (Vuitton, Chanel, Dior, Hermès). Il s'agit d'un phénomène que l'on retrouve dans plusieurs quartiers à Shanghai notamment à Xujiahui. 
Illustration 1 - La place du Peuple et la rue de Nankin (Ouest)

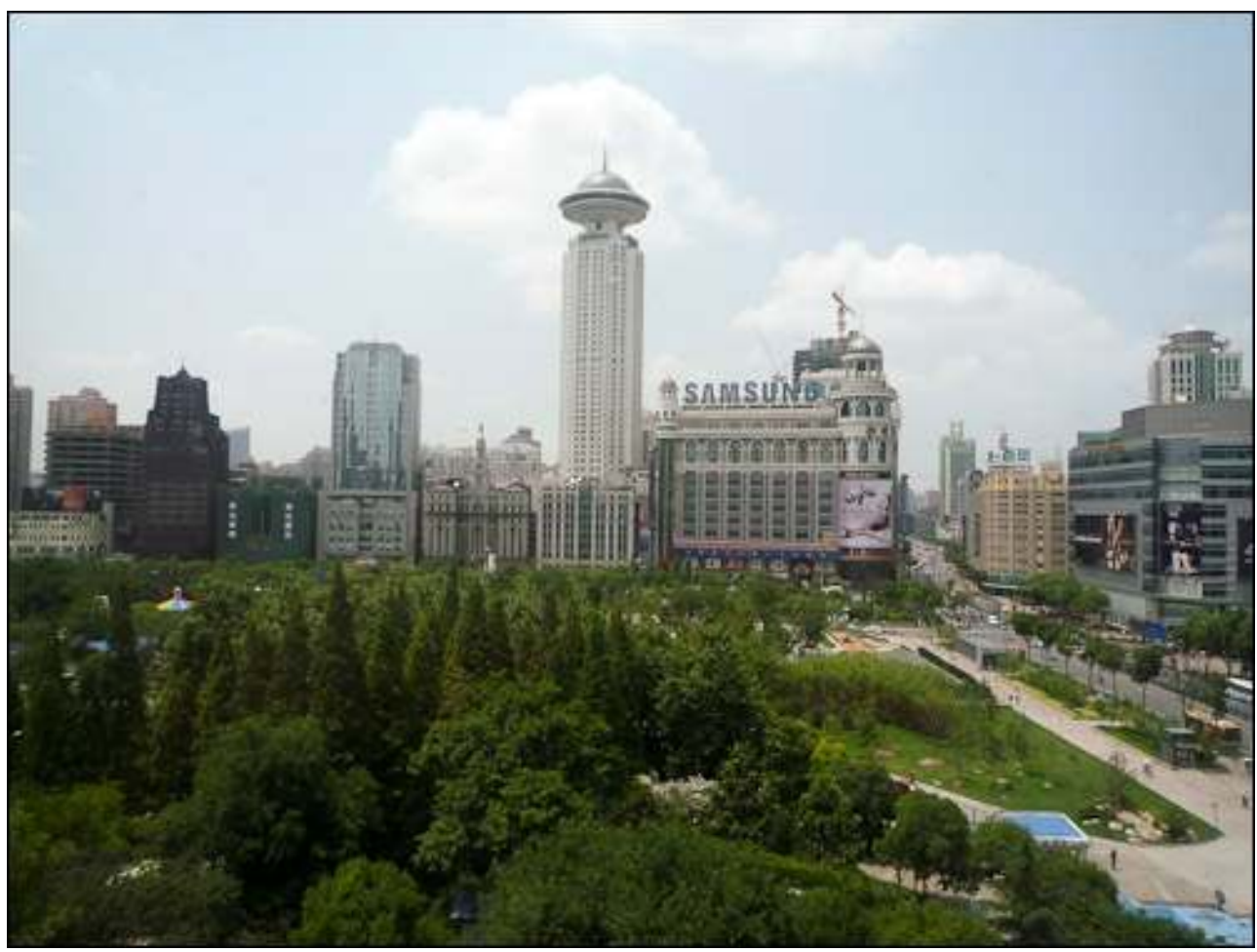

Cliché pris depuis le musée de l'urbanisme.

Source : Howard Vazquez, juin 2009.

- La rue Huaihai (Huaihailu), longue de près de 6 kilomètres, se compose d'un secteur est (Huaihaidonglu), d'un secteur central (Huaihaizhonglu) et d'un secteur ouest (Huaihaixilu). Dans son ensemble, la rue Huaihai est une rue commerciale animée qui s'étend de l'arrondissement de Luwan jusqu'à celui de Xuhui. La partie orientale, lieu des résidences officielles, concentre principalement des immeubles et des bureaux. La partie centrale, large voie commerciale bordée d'arbres, se compose de restaurants, d'hôtels, de théâtres et de magasins. Dans ce secteur, la rue est bordée par des boutiques de luxe des grandes marques internationales (Calvin Klein, Hugo Boss, Gucci) et des centres commerciaux modernes. Enfin, le secteur ouest (Huaihaixilu), objet de cette étude, est à la fois une zone commerciale, où se regroupent les marques chinoises et du monde entier, et un centre d'affaire voué à une tertiarisation des activités (bureaux, administration, banques, assurances). 
Illstration 2 - La rue Huaihai (Ouest)

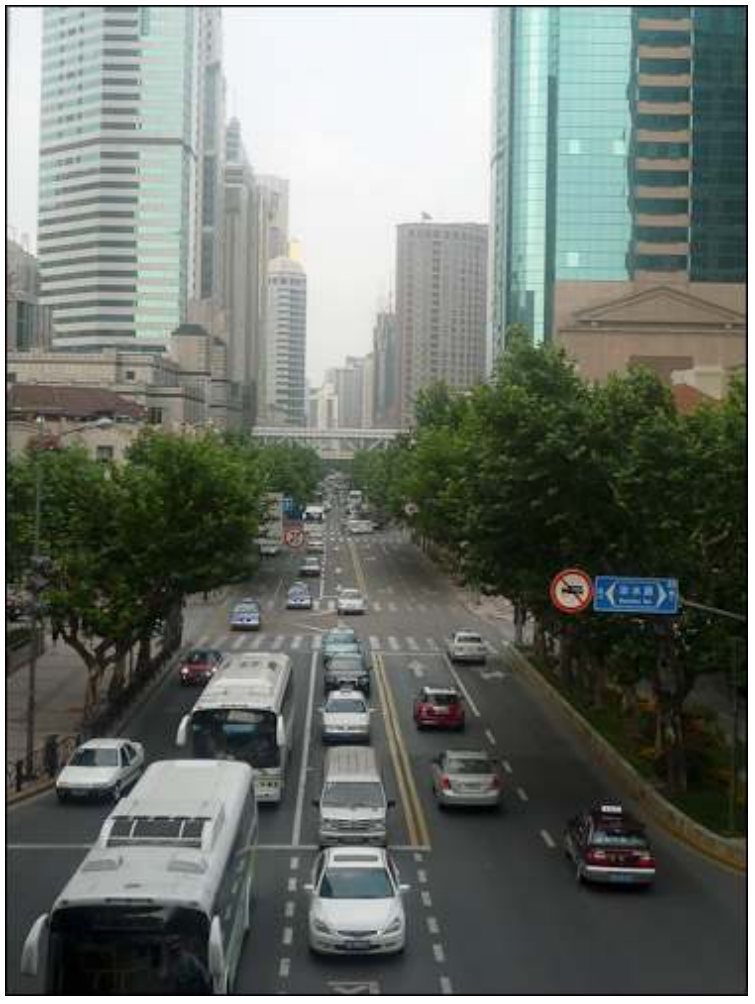

Source : Howard Vazquez, juillet 2009

- La rue du Sichuan dans son secteur nord (Sichuanbeilu), centre de l'arrondissement de Hongkou, est un axe commerçant, long de 3,6 kilomètres, qui s'étend du parc Lu Xun (Lu Xun gongyuan) au nord jusqu'au cours d'eau de la rivière Suzhou, principal affluent du Huangpu, plus au sud. Il s'agit d'une rue résidentielle et commerciale, composée de services et d'équipements, qui rivalise très difficilement avec les rues de Nankin (Ouest) et Huaihai (Ouest). Localisée en partie dans l'ancienne concession internationale, la rue du Sichuan (Nord) conserve les héritages des années 1920 et 1930 de l'ancien Shanghai. 
Illustration 3 - La rue du Sichuan dans son secteur nord

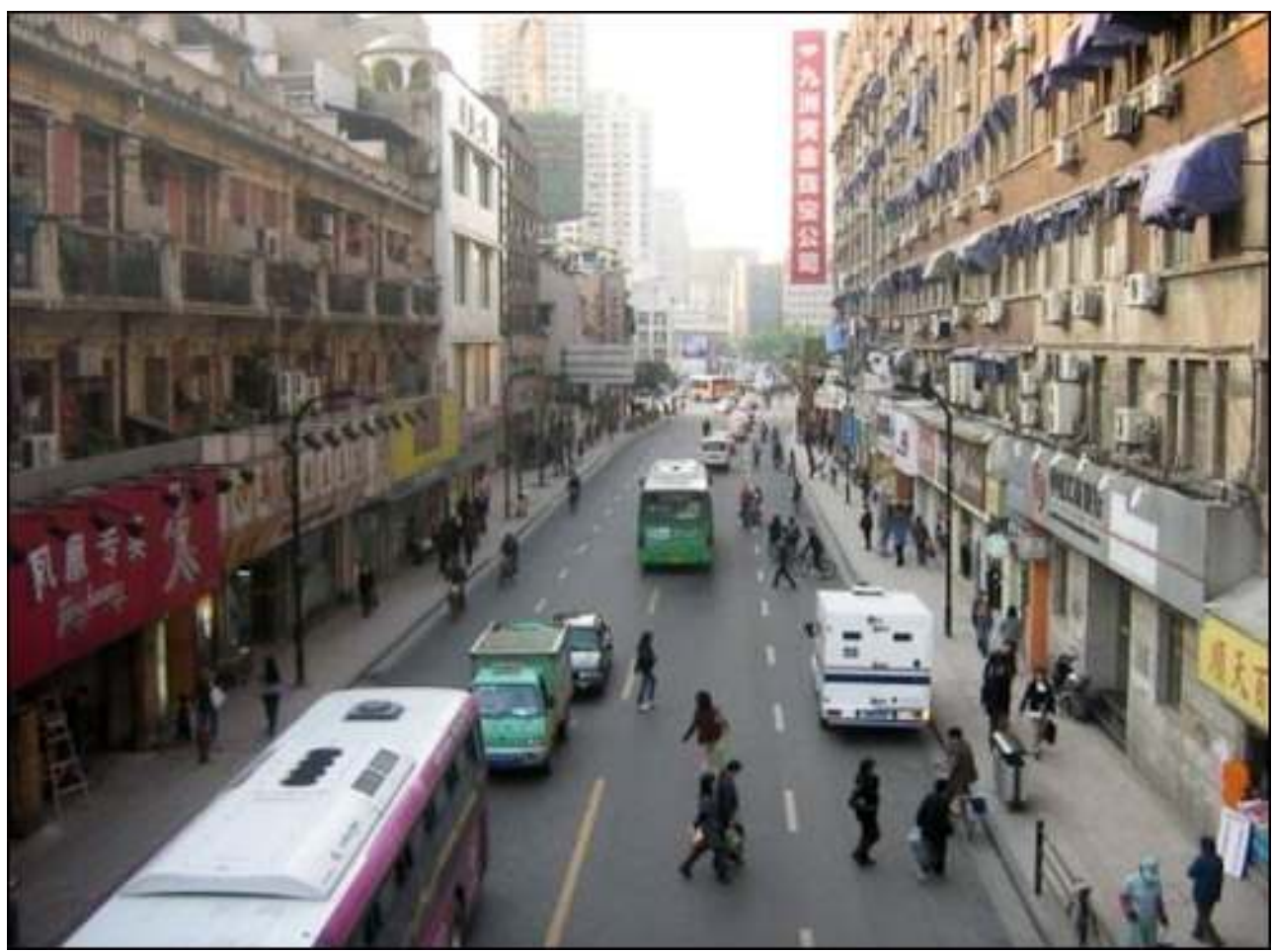

Source: Howard Vazquez, mars 2008

Les artères péri-centrales sont des voies majeures qui constituent en quelque sorte les grands boulevards de Shanghai. Ce sont de longues et larges artères, à la fois commerciales et résidentielles, localisées dans les arrondissements urbains centraux de Shanghai. En définitive, les artères péri-centrales sont des rues qui permettent d'obtenir une représentation et une image emblématique de Shanghai. En effet, selon Jérôme Monnet ${ }^{5}$ : «la représentativité d'une rue se fonde paradoxalement sur son exceptionnalité dans la ville ».

\section{Des rues historiques}

12 L'ordonnancement des rues de Shanghai se distingue de celui de Pékin. Les rues de Shanghai sont plus étroites et sinueuses que celles de Pékin. Les héritages d'une ville tripartite datant de l'époque des concessions étrangères contribuent largement à la morphologie urbaine actuelle. De facto, les artères péri-centrales de Shanghai sont des rues très longues en relation directe avec le centre-ville historique. Les héritages urbains sont multiples.

- La rue de Nankin (Ouest) constitue l'ancienne rue de divertissement de Shanghai. Cet axe était alors un complexe de récréation composé de restaurants, cabarets, dancings, cinémas, salles de jeux et de spectacles (le grand cinéma, le Majestic, le Metropole). Le Paramount, situé dans la partie ouest de la rue, était alors le plus célèbre dancing des années 1920. De même, le Parc Hotel, érigé en 1933, demeura longtemps le plus haut gratte-ciel de Shanghai (86 mètres). La rue de Nankin dans son tronçon oriental, davantage consacrée aux finances et aux services, était alors la rue commerciale la plus animée de Shanghai ${ }^{6}$. Depuis les berges du Huangpu, la trame viaire de la rue de Nankin constituait un axe de circulation incontournable composé d'infrastructures urbaines majeures de la métropole portuaire. 
Figure de la ville, le Bund concentre les édifices historiques datant de la fin du XIXe siècle jusqu'aux années 1940.

- La rue Huaihai, ancienne avenue Xijianglu, rebaptisée avenue Joffre pendant la Première Guerre mondiale, était la rue des élégances, considérée comme les Champs-Élysées de Shanghai ${ }^{7}$. Artère principale de l'ancienne concession française, cette rue était bordée de restaurants, de cafés et de boutiques de mode. Les années 1917-1918 marquent l'essor de la culture commerciale de Shanghai, et l'installation des premiers grands magasins ${ }^{8}$. La rue Huaihai était alors la vitrine commerciale de la mode occidentale.

- La rue du Sichuan (Nord), centre de l'arrondissement de Hongkou, située en partie dans la concession internationale, se caractérise par ses héritages urbains. Dans les années 1920, les membres fondateurs de la Ligue des écrivains de gauche vécurent dans la rue piétonne et adjacente de Duolun (Lu Xun et Ye Shentao). En 1937, l'occupation de la rue du Sichuan (Nord), ainsi que l'ensemble de l'arrondissement de Hongkou, par l'armée japonaise, contribua au caractère historique et culturel de la rue. Alors nommée Tong Jan Yan, la rue du Sichuan (Nord) était le symbole de la résistance chinoise. Troisième axe commercial de Shanghai, après les rues de Nankin et Huaihai, la rue du Sichuan (Nord) possédait un rayonnement économique plus limité.

13 Ces trois rues sont des espaces marqués par le poids des héritages urbains. Ce sont des axes privilégiés et des éléments structurants de la ville. En dépit de la modernisation rapide de Shanghai, la hiérarchie urbaine des artères péri-centrales est toujours respectée. Les rues Huaihai (Ouest et Est) et de Nankin (Ouest et Est) se caractérisent par des transformations urbaines et sociales plus rapides que celle du Sichuan (Nord). La dimension morphologique et les fonctions anciennes de ces rues expliquent leurs caractéristiques actuelles.

\section{Des espaces publics multifonctionnels}

Les artères péri-centrales sont animées par une série de flux de circulation. Il s'agit de rues bruyantes à usage obligatoire fréquentées par les riverains et les passants à différentes échelles temporelles. Ce sont des rues résidentielles et commerciales qui se caractérisent par une intensité des flux de mobilité aux heures de pointes (de $7 \mathrm{~h}$ à $9 \mathrm{~h}$ le matin, de $17 \mathrm{~h}$ à $20 \mathrm{~h}$ le soir). Les pics de circulation, le matin et le soir, s'expliquent par les mouvements pendulaires entre le domicile et le lieu de travail. Même si Shanghai compte officiellement près d'un million de véhicules en $2000^{9}$, ce qui est relativement peu pour une métropole de cette taille, l'augmentation du nombre de voitures dans l'ensemble de l'agglomération affecte directement l'organisation de ces rues. Devant la densification du trafic, l'élargissement de la voirie est devenu nécessaire. Les projets urbains se sont très largement focalisés sur l'élargissement progressif de ces voies d'accès jusqu'au centre-ville. En termes d'équipements, les artères péri-centrales sont des rues abondamment desservies par les moyens de transport. Les réseaux de bus et de métros sont directement reliés au centre-ville, ce qui contribue largement au dynamisme économique de ces rues. Dès lors, la construction de nouveaux équipements de transport ne cesse de se multiplier près de ces axes (construction de la ligne de métro $n^{\circ} 9$ dans la rue du Sichuan (Nord)).

Par leur animation et leur mise en scène de la vie quotidienne ${ }^{10}$, ces rues peuvent être qualifiées de banales. Les interactions entre l'environnement social et les usages des services publics et privés (commerces, bureaux, écoles, hôpitaux, bureaux de poste, 
banques, assurances) contribuent à la vitalité des rues. Les équipements (transports, parcs, jardins), les activités (commerces, bureaux) et l'achat de biens de première nécessité participent pleinement à la riveraineté des artères péri-centrales.

En termes d'activités, il s'agit de trois rues commerçantes à spécialisation récréative. Les activités offertes sont variées et ouvertes à la société de consommation : magasins, centres commerciaux, cinémas, karaokés. Le caractère commercial et récréatif de ces rues crée une relation obligée avec le mouvement ${ }^{11}$. La rue en tant qu'espace public est le dernier espace franchi avant d'entrer dans le domaine privé. Dès lors, les pratiques citadines font des artères péri-centrales un espace complexe, avec des trajectoires et des temporalités multiples.

\section{Les artères péri-centrales, des rues qui participent activement à la vie de la métropole}

17 La rue est l'essence même de l'urbain. Les artères péri-centrales font l'objet d'intenses mutations urbaines et sociales. L'augmentation constante de la population de Shanghai nécessite davantage de logements, de services, d'équipements et de modes de déplacement. Devant ce constat, le gouvernement local exprime ses ambitions. Les bénéfices pouvant être engendrés encouragent les motivations de la municipalité.

Les artères péri-centrales se caractérisent par une double vocation :

- d'une part, redonner une image attractive à la rue ;

- et d'autre part, ramener vers ces rues, une population aisée ou des activités tertiaires, peu coûteuses en aides sociales et pourvoyeuses de rentrées fiscales. Ce processus de gentrification c'est-à-dire la transformation d'un quartier modeste ou déshérité qui, par l'intermédiaire d'une réhabilitation immobilière, voit s'installer une population plus aisée affecte directement les artères péri-centrales de Shanghai.

\section{Des rues en régénération urbaine}

19 Ces rues sont des éléments de composition urbaine indispensable à la vie de la métropole chinoise. Le bâti urbain qui borde la voie est hétéroclite. Il se compose à la fois de tours modernes d'habitation, de centres commerciaux, de bureaux d'affaires et d'habitats collectifs chinois.

Les opérations urbaines visant à transformer les artères péri-centrales se sont multipliées. Les lilong, c'est-à-dire l'habitat collectif typiquement shanghaïen organisé en courées, et articulé en un réseau de passages et de ruelles convergeant vers les rues principales, sont progressivement détruits ${ }^{12}$. Le dépérissement relatif des lilong, composés en bois et en briques, sur deux étages, encourage les opérations immobilières. Entre les années 1991 et 2000, 26 millions de mètres carrés de vieilles maisons ont été détruites dans tout Shanghai et 660000 ménages relogés ${ }^{13}$. Le remplacement des anciennes strates urbaines shanghaiennes par des tours modernes est comparable à la rénovation urbaine de Pékin pour l'organisation des Jeux olympiques. De facto, l'Exposition universelle de 2010 accélère les transformations urbaines de Shanghai. 
Illustrations 4 et 5 - La persistance des anciennes strates de la société chinoise
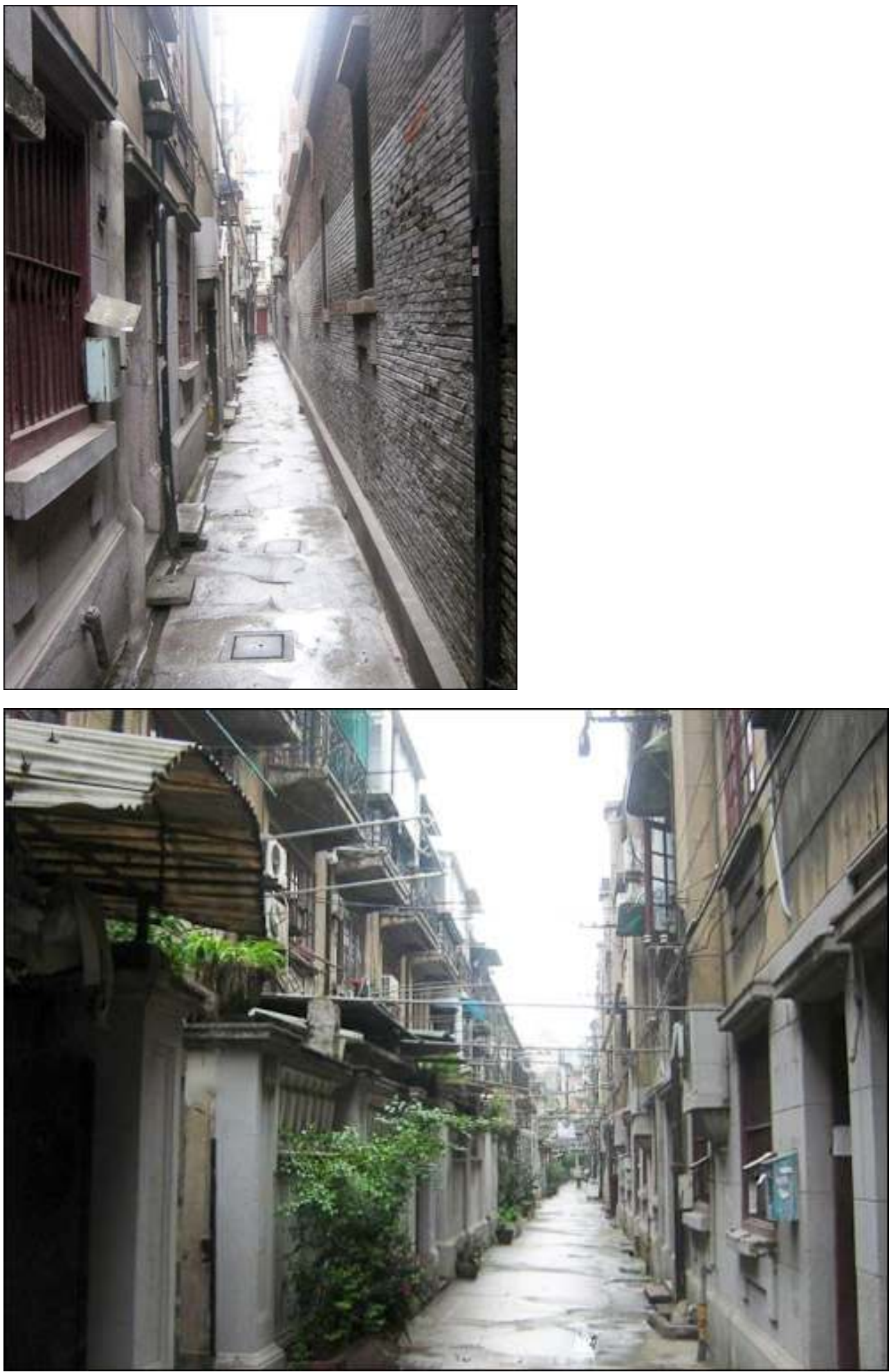

Clichés pris dans les lilong de la rue du Sichuan (Nord).

Source : Howard Vazquez, avril 2008

21 Mais, malgré la modernisation actuelle, les structures urbaines des artères péricentrales restent les mêmes. En effet, la construction de nouveaux immeubles ne 
détruit pas complètement l'environnement urbain mais le modernise. On distingue deux types d'opérations :

- d'une part, la rénovation urbaine, à l'image de la rue Huaihai (Ouest) et celle de Nankin (Ouest), qui se traduit par la destruction de l'ancien tissu urbain et la mise en place d'immeubles neufs construits dans le cadre d'opérations immobilières ;

- d'autre part, la réhabilitation qui consiste à remettre en valeur des quartiers dégradés par la restauration de façades et des logements. La rue du Sichuan (Nord) s'inscrit davantage dans cette politique de réhabilitation de la voirie.

L'habitat collectif de faible hauteur laisse place à des immeubles modernes de grande taille. Dès lors, l'environnement urbain actuel se compose à la fois d'immeubles ultramodernes et de petits immeubles de deux ou trois étages où vivent plusieurs familles. Le contraste entre les tours modernes et éclairées la nuit et les strates précédentes de la société chinoise montre toute l'ambiguïté de la modernisation urbaine de Shanghai.

Illustration 6 - La destruction et le remplacement de l'ancien tissu urbain par des tours d'habitations et des immeubles modernes

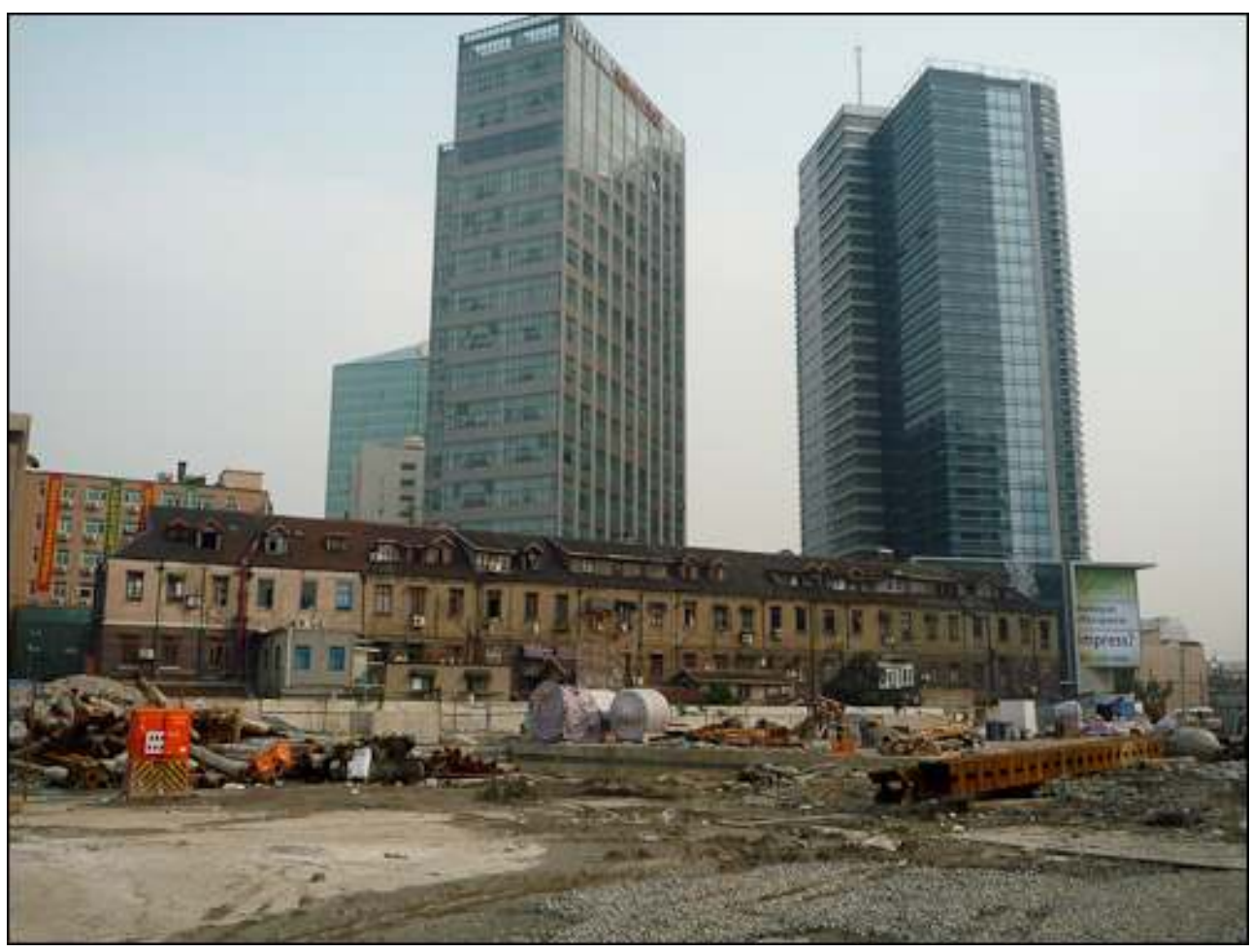

Cliché pris dans la rue du Sichuan (Nord).

Source : Howard Vazquez, juin 2009

Shichen, un jeune étudiant raconte ${ }^{14}$ :

Quand j'étais enfant, il y a avait des lilong dans tout l'arrondissement de Hongkou. Dans la rue du Sichuan (Nord), il n'y avait que des maisons basses chinoises. Et, progressivement, on les a détruites. Aujourd'hui, le gouvernement décide de construire de plus en plus de tours. Désormais, l'immobilier explose, les logements coûtent extrêmement cher, si bien que nous ne pouvons plus vivre ici...

Les artères péri-centrales apparaissent clairement comme un outil de valorisation foncière. Les promoteurs immobiliers et la Municipalité de Shanghai multiplient les projets urbains. De nombreux immeubles sont actuellement en construction. Cette 
rénovation urbaine s'accompagne généralement d'une hausse du prix des loyers et du foncier. Globalement, une transition entre rue résidentielle et rue commerciale s'est amorcée. La part des résidents se réduit au profit des activités commerciales. De nombreux riverains ont été contraints et forcés de déménager vers les arrondissements périphériques. Même s'il ne s'agit pas là d'un phénomène typiquement shanghaïen, le Shanghai de 2010, qui détruit ses lilong et expulse une large partie de ses populations en périphérie, s'inscrit dans la continuité avec les années $1920^{15}$.

Face à ce constat, le développement actuel de Shanghai se concrétise par les espaces périphériques. Désormais, de plus en plus de Shanghaiens résident dans les arrondissements péri-urbains. Le développement des villes nouvelles, situées dans les marges urbaines, est censé désengorger le centre-ville historique. Outre la ville-centre de Shanghai, le $\mathrm{XI}^{\mathrm{e}}$ plan quinquennal propose une structuration fondée sur trois villes principales (Songjiang, Jiading et Luchaogang), dix villes nouvelles, 60 bourgs et 600 villages ${ }^{16}$. De même, les grandes compagnies étrangères désireuses d'implanter leurs locaux à Shanghai investissent ces espaces. Le prix du mètre carré, plus attrayant, explique ces nouvelles dynamiques territoriales. En termes de dynamiques urbaines, les artères péri-centrales apparaissent clairement comme des rues en intenses mutations. Une des conséquences des politiques de rénovation et réhabilitation des rues est l'apparition de nouvelles nuisances urbaines. Parfois au bord de l'asphyxie, ces voies sont confrontées à de nouvelles nuisances: pollution, engorgement, embouteillage, bruit, luminosité. La vie quotidienne des riverains est directement affectée.

Mme Liang, une retraitée raconte ${ }^{17}$ :

Les nouvelles tours empêchent la circulation de l'air et obstruent la luminosité des riverains qui résident dans les lilong. Il ne faut pas penser uniquement au confort des gens qui vivent dans les tours modernes. Il faut prendre en compte l'ensemble des riverains.

\section{Les artères péri-centrales, support de la gentrification} dynamiques sociales. En effet, les artères péri-centrales sont des espaces d'interactions sociales majeures. La rue en tant qu'espace de frottements et d'affrontements ${ }^{18}$ peut se définir comme une réalité économique et sociale. Les artères péri-centrales sont des rues résidentielles. Face à ce constat, trois types de résidents peuvent être distingués :

- Les anciens habitants qui sont nés et ont grandi dans le quartier. Ils ont un lien particulier avec l'espace. Généralement, leurs parents résidaient déjà dans ces rues.

- Les riverains qui se sont installés en masse dans les années 1970 et 1980.

- La troisième catégorie concerne les nouveaux arrivants, issus de la classe supérieure, qui investissent ces rues depuis la deuxième moitié des années 2000.

Le profil socio-géographique des riverains se compose à la fois de personnes modestes qui résident dans les lilong, la plupart d'entre eux gagnent entre 1200 et 1500 yuans par mois, et une catégorie sociale supérieure qui contribue à la privatisation de l'espace bâti. Ces derniers investissent généralement les nouveaux appartements situés dans les tours modernes. Les artères péri-centrales se caractérisent par une relative mixité sociale. Si classe aisée, classe moyenne et classe modeste se côtoient, ces propos sont une nouvelle fois à nuancer. En Chine, on s'oriente de plus en plus vers une privatisation de l'espace urbain. Les anciens riverains sont remplacés par une nouvelle 
catégorie sociale d'individus. Les artères péri-centrales voient leur population diminuer lentement, et l'une des conséquences des politiques de rénovation urbaine est la gentrification de l'espace. Il s'agit d'une gentrification forte, rapide et ségrégative. Les classes moyennes ${ }^{19}$ et inférieures quittent les artères péri-centrales pour aller vivre dans les arrondissements péri-centraux. Dès lors, les anciens riverains sont remplacés par des employés, des cadres et professions intellectuelles supérieures. Ce processus de stratification socio-spatiale affecte directement les artères péri-centrales de Shanghai.

Cependant, ce processus de gentrification ne touche pas les artères péri-centrales de façon homogène. En effet, la stratification socio-spatiale des rues Huaihai (Ouest) et de Nankin (Ouest) est plus forte que dans celle du Sichuan (Nord). Le caractère résidentiel de l'arrondissement de Hongkou explique en partie la lenteur du processus. La gentrification n'est pas encore achevée. Mais, devant la montée des prix du foncier et la hausse des loyers, l'environnement social de la rue du Sichuan (Nord) risque de changer très rapidement.

Une part non négligeable de migrants provinciaux, installés à Shanghai dans l'espoir d'obtenir un emploi et épargner assez d'argent pour acheter une maison dans leur province d'origine, résident dans les artères péri-centrales. Cependant, on constate de plus en plus que les migrants tendent à rester à Shanghai et à faire venir leur famille. Dans la rue du Sichuan (Nord), près de $22 \%$ des riverains ne sont pas natifs de Shanghai ${ }^{20}$. La ville de Shanghai a toujours été une ville de migrants. Par la notion de natif, nous considérons ici, les personnes qui ont un permis permanent de résidence et non pas ceux qui sont nés à Shanghai mais dont les parents venaient d'une autre province.

32 Le cas de la rue du Sichuan (Nord) est à replacer dans le contexte de l'arrondissement de Hongkou. Il s'agit d'une rue résidentielle, occupée par une large classe moyenne. Dès lors, l'expulsion des résidents est beaucoup plus difficile. Et les dépenses pour reloger les habitants vers les villes nouvelles sont beaucoup plus coûteuses pour le gouvernement shanghaien. Dans le secteur de la rue du Sichuan (Nord), le gouvernement concentre la plupart des projets d'aménagement sur l'axe commercial. Selon Zhong Yiqun, une ouvrière ${ }^{21}$ :

Nos maisons sont détruites, les toits sont en train de s'écrouler, il y a des fuites, des moisissures et des rats. Ces bâtiments ont été construits dans les années 1970 et, à présent, il faudrait les rénover. Le gouvernement concentre tous les efforts de rénovation dans la rue du Sichuan (Nord). Les rues adjacentes sont laissées à l'abandon et le développement en façade de la rue du Sichuan (Nord) ne se poursuit pas dans les rues parallèles...

À l'inverse, les rues de Nankin (Ouest) et Huaihai (Ouest), ainsi que l'ensemble des arrondissements de Luwan, Jing'an et Xuhui se caractérisent par des transformations socio-spatiales très rapides. Les dynamiques sociales et paysagères sont très soutenues et beaucoup plus radicales. Les nouveaux logements situés dans les tours d'habitation modernes sont occupés par les classes sociales supérieures. Les artères péri-centrales sont des axes commerçants, dynamiques, où les offres professionnelles tendent à se multiplier, ce qui permet notamment aux riverains d'obtenir un emploi près de leur logement.

L'aspect récréatif des artères péri-centrales contribue au caractère passant des rues. Les artères péri-centrales sont des rues très fréquentées en semaine et le week-end. Près de $52 \%$ des personnes interrogées ont une pratique quotidienne ${ }^{22}$ de la rue du 
Sichuan (Nord). Globalement, toutes les tranches d'âges de la population shanghaienne fréquentent les artères péri-centrales. Cependant, le développement des arrondissements péri-urbains réduit de façon significative la pression démographique et la fréquentation passante du centre-ville. En termes de pratiques citadines, les artères péri-centrales sont des lieux d'interaction sociale. Jeunes cadres dynamiques, couples, groupes d'amis, retraités fréquentent en nombre ces lieux de vie. Ce sont des rues très fréquentées par les étudiants. Ainsi, dans la rue du Sichuan (Nord), l'animation étudiante s'accroît à mesure que l'on s'approche des Universités Tongji et Fudan.

Même si le profil socio-géographique des passants présente de nombreuses similarités, plusieurs distinctions apparaissent entre les artères péri-centrales. La rue de Nankin (Ouest) et celle de Huaihai (Ouest), principales artères commerciales de la ville, sont le théâtre des transformations sociales. Les riverains sont majoritairement des gens issus des catégories sociales supérieures. Ils fréquentent, pour la plupart, les boutiques de luxe et les centres commerciaux ultra-modernes. Même si la gentrification est d'abord résidentielle, nous pouvons employer le terme de "gentrification passante» pour décrire le caractère passant des rues. Il s'agit du processus de transformation d'une rue ou d'un quartier modeste qui, par l'intermédiaire du taux de fréquentation des classes supérieures et d'une certaine pratique des promeneurs, orientée vers les produits coûteux, voit l'environnement social de la rue se modifier. À l'inverse, la rue du Sichuan (Nord) est une rue populaire, composée d'un faible nombre de magasins de standing. Dès lors, la plupart des passants appartiennent aux classes sociales moyennes et inférieures. Près de $72 \%$ des promeneurs vivent dans l'arrondissement de Hongkou, et respectivement $5 \%$ et $8 \%$ des passants vivent dans les arrondissements limitrophes de Zhabei et de Yangpu. En définitive, la rue du Sichuan (Nord) est un axe commercial fréquenté majoritairement par les locaux.

Selon Zhang, un étudiant de l'Université Tongji ${ }^{23}$ :

La rue du Sichuan (Nord), c'est juste un endroit pour les locaux, pour les vrais Shanghaiens qui ont toujours vécu dans l'arrondissement de Hongkou. Il n'y a pas de grands magasins comme dans la rue de Nankin (Est) et celle de Huaihai. C'est vraiment la classe populaire. Le marché de la contrefaçon de Qipulu, situé dans la partie sud de la rue, symbolise l'environnement social de la rue du Sichuan (Nord). Il n'y a que des petites boutiques et des petits restaurants...

\section{Selon Xu, un informaticien ${ }^{24}$ :}

La plupart des gens ne disent pas qu'ils vont faire du shopping dans la rue du Sichuan (Nord), ça ne se dit pas à Shanghai! Ce n'est pas assez à la mode. Le gouvernement local n'a pas encore réussi à attirer les foules dans cette rue...

De même, une autre réalité sociologique se dessine dans les artères péri-centrales. Une observation in situ des rues fait apparaître un autre caractère de la "gentrification passante ». La fréquentation des rues par la population dite marginale ou marginalisée fait apparaître plusieurs distinctions. La rue Huaihai et celle de Nankin sont des rues où des dispositifs envers les indésirables (SDF, mendiants) sont mis en place. Même si l'on voit tout de même les mendiants faire les poubelles et faire la manche dans ces rues, la police veille à rendre invisibles les sans-logis pour ne pas nuire à l'image des rues, et effrayer les touristes et les classes sociales supérieures. Néanmoins, les mesures de contorsion de l'espace public shanghaien sont plus brutales et pas encore aussi subtiles qu'à Paris (arrosage inutile des parcs publics, remplacement sous l'alibi design des bancs du métro parisien ${ }^{25}$ ). La "gentrification passante» des rues est donc entretenue. La 
valorisation d'une rue peut donc se faire par la composition sociale des promeneurs. La présence de populations riches fortement ancrées dans la rue est un facteur décisif dans le processus de "gentrification passante ». À l'inverse, la rue du Sichuan (Nord) est une rue populaire où une part significative de sans-logis est largement visible. Dès lors, les efforts consentis pas le gouvernement local ne sont pas comparables avec les rues de Huaihai et Nankin.

Chen Lei, un jeune cadre raconte ${ }^{26}$ :

Il y a beaucoup trop de sans-logis dans la rue du Sichuan (Nord). Ils veulent qu'on leur donne de l'argent sans qu'ils fassent d'efforts. Ils portent des vêtements sales. Ils demandent un yuan aux passants. Je ne pense pas que ce soit une bonne chose. Ça donne une très mauvaise image aux étrangers. Dans la rue de Nankin et celle de Huaihai, la police fait un effort pour les rendre invisibles, notamment parce que les touristes et les étrangers vont y faire leurs achats. Mais, ici, dans la rue du Sichuan (Nord), la police ne leur dit rien. Ils leur laissent tout faire!

41 Face à ce constat, les artères péri-centrales de Shanghai sont des rues qui subissent des dynamiques urbaines et sociales très rapides, qui participent pleinement à la vie de la métropole chinoise.

\section{Des rues vecteurs de la mondialisation}

Si les artères péri-centrales sont des rues attractives qui participent à la vie de la ville, il n'en demeure pas moins qu'elles participent aussi activement au processus de mondialisation.

\section{Des rues attractives à la recherche de rayonnement international}

Les artères péri-centrales bénéficient d'une attractivité et d'un dynamisme économique. Le terme d'attractivité désigne ici la capacité d'une rue à attirer des commerces de luxe, des touristes, des cadres et des professions intellectuelles supérieures. Par le marketing urbain, la ville cherche à promouvoir les artères péricentrales.

Ming, un jeune commercial raconte ${ }^{27}$ :

Il existe trois grandes rues à Shanghai : la première est la rue de Nankin dans son secteur est et ouest. Il s'agit d'une rue commerciale très touristique. La seconde est la rue Huaihai. Cette rue concentre des boutiques de luxe et les sièges des grandes entreprises. La dernière est la rue du Sichuan (Nord). C'est une rue beaucoup moins dynamique que les deux précédentes. C'est une rue populaire...

Ces rues concentrent des fonctions économiques, culturelles et de loisir. Les artères péri-centrales sont des rues animées et commerçantes à spécialisation récréative. Elles se composent de bureaux, d'activités de haut niveau ou à la gestion des affaires bureaucratiques. Par leur nature, ces rues sont des lieux de concentration d'activités publiques et privées : bureaux, banques, assurances, sièges de sociétés, immobilier, télécommunication. La spécialisation économique des activités se caractérise par une tertiarisation des activités. La part des activités tertiaires est passée de $30 \%$ à $53 \%$ du PIB municipal entre 1990 et $2007^{28}$. Ainsi, les docks et les anciennes usines situés le long de la rivière Suzhou, près de la rue du Sichuan (Nord), sont promis à des réaménagements touristiques. Les grands écrans de télévision et les gigantesques panneaux publicitaires ont remplacé les magasins de vêtements et les petites boutiques 
de produits de première nécessité. Le commerce de proximité, encore présent dans les années 1980, a laissé place à des centres commerciaux modernes et à la consommation de masse. Les activités offertes sont variées et ouvertes à la société de consommation. la rue est absorbée par l'envie de consommer mais aussi par l'envie uniformisée du paraître et du désir, surtout chez les jeunes et les cadres supérieurs, qui sont des acteurs majeurs de ces nouvelles pratiques urbaines. De même, dans les rues de Nankin (Ouest) et de Huaihai (Ouest), le commerce informel tend progressivement à disparaître, alors que dans la rue du Sichuan (Nord) le commerce informel est encore une composante hautement visible. Les étalages présentent des produits divers: montres, bijoux, cigarettes vendues à l'unité, cartes téléphoniques, crickets ; on trouve également des activités de services : réparations de chaussures, de vélos, de parapluies. La rue du Sichuan (Nord) se distingue de la rue de Nankin (Ouest) et celle de Huaihai par la volonté de protéger son environnement social et urbain. Il s'agit plutôt d'une rue populaire vouée à des activités sportives, culturelles et artistiques. D'ailleurs, sa modernisation est à présent combinée à une politique patrimoniale en lien avec une rue adjacente et piétonnière, la rue Duolun ${ }^{30}$. 


\section{Des sociabilités quotidiennes menacées}

50 Au-delà de l'homogénéisation des pratiques citadines et des modes de vie, les artères péri-centrales sont à la fois de réels espaces publics au sens sociologique du terme. La rue shanghaienne, en tant qu'espace de sociabilité, se caractérise par un recul progressif du lien social. Les relations de solidarité entre les riverains tendent à se restreindre. Socialement, une rupture est observable. Après la rénovation urbaine, les liens qui unissent les riverains ont tendance à disparaître. Les mutations urbaines et sociales expliquent en partie cette orientation vers l'anomie. Dès lors, de nouveaux comportements sociaux apparaissent.

Yuan, un employé de magasin raconte ${ }^{31}$ :

Je pense que les transformations de l'environnement social et paysager nous conduit vers de nouveaux comportements. Aujourd'hui, je peux faire appel à mon voisin. Mais, le jour où tout le monde aura son appartement, les gens ne se parleront plus. On pourra rester dix ans, l'un à coté de l'autre, sans se connaître...

52 Avec la destruction progressive des lilong, c'est l'ensemble des lieux de vie de la communauté résidentielle qui disparaît. Les gens ne s'assoient plus devant leur maison, ne se rassemblent plus sur les trottoirs pour regarder la télévision. L'arrivée de l'air conditionné dans les maisons privées explique en partie le recul de cette pratique. Les chants, les activités artistiques, le commerce informel tendent à disparaître progressivement. Les vendeurs ambulants n'installent plus leurs tables sur les trottoirs. De même, les dialectes autrefois parlés dans certains arrondissements, et que seuls les vrais Shanghaiens pouvaient détecter, ont disparu. En se modernisant, les artères péricentrales ont tendance à perdre leur identité chinoise et à s'universaliser en suivant des intensités et des modes différents.

\section{Conclusion}

53 Au terme de cette analyse de trois artères péri-urbaines de Shanghai, les dynamiques à la fois sociales, politiques et économiques qui sont en jeu suivent le rythme soutenu de l'internationalisation des économies. Les artères péri-centrales de Shanghai sont des rues soumises à des mutations urbaines et sociales très intenses. Les mutations de trois artères péri-urbaines révèlent les transformations observées à l'échelle de l'ensemble de la ville dans le cadre de la redéfinition plus globale de la municipalité. Appréhender concrètement l'évolution actuelle des artères péri-urbaines de Shanghai permet de donner des éléments de réflexion sur la maîtrise de leur évolution depuis le début des années 1990. La rue de Nankin (Ouest) se caractérise par des dynamiques urbaines et sociales très rapides et ségrégatives. Cette artère péri-urbaine se compose de centres commerciaux modernes, de gratte-ciel, de bâtiments officiels et d'immeubles de bureaux. La rue Huaihai (Ouest) se caractérise aussi par des dynamiques urbaines et sociales très intenses. On peut réellement parler de stratification socio-spatiale. Il s'agit d'une vitrine commerciale pour la ville de Shanghai. Les services (commerces, bureaux) et équipements (administration) y sont très nombreux. Enfin, la rue du Sichuan (Nord) se définit par des dynamiques urbaines et sociales plus lentes. Il s'agit d'un axe commerçant à caractère résidentiel. C'est une rue populaire où les anciennes strates de la société chinoise sont hautement visibles. L'évolution morphologique, fonctionnelle et sociale des rues participent pleinement à la vie de la métropole et au processus de 
mondialisation. Cependant, en se modernisant, ces rues symbolisent aussi la perte d'une certaine identité chinoise dans la ville. En intégrant des produits de standing, ces rues perdent leurs singularités. Les transformations des artères péri-urbaines suivent celle de la société chinoise. La question reste ainsi de trouver un équilibre acceptable et accepté entre la modernisation des rues et la préservation des particularités locales.

\section{BIBLIOGRAPHIE}

Bergère M.-C., 2002. Histoire de Shanghai. Paris, Fayard, 520 p.

Brès A., 2005. Inscription territoriale des mobilités et riveraineté des voies. Thèse d'urbanisme et d'aménagement, Université Paris 1 Panthéon-Sorbonne.

Brès A., mars 2007. De la voirie à la riveraineté et attribution des stratégies d'inscription territoriale des mobilités périurbaines. Flux, $\mathrm{n}^{\circ}$ 66-67, p. 87-95.

Brès A., octobre-Décembre 1998. Le système des voies urbaines : entre réseau et espace. Flux, $\mathrm{n}^{\circ}$ 34 , p. 4-20.

Charmes E., 2006. La rue, village ou décor ? : Parcours de deux rues de Belleville. Paris, Creaphis, 157 p.

Choay F., Merlin P., 2005. Dictionnaire de l'urbanisme et de l'aménagement. Paris, Presses Universitaires de France, $964 \mathrm{p}$.

Damon J., 1993. Les Indésirables dans les espaces de transport. Les exemples de la RATP et de la SNCF. Recherche effectuée pour le compte de la direction des Grandes Lignes SNCF, sous la direction d'Hervé Laroche, ESCP, 92 p.

Fleury A., 2007. Les espaces publics dans les politiques métropolitaines. Réflexions au croisement de trois expériences : de Paris aux quartiers centraux de Berlin et Istambul. Thèse de géographie, Université Paris 1 Panthéon-Sorbonne, 693 p.

Goffman E., 2007. La Mise en scène de la vie quotidienne, Paris, Éditions de minuit, 256 p.

Gourdon J.-L., 2001. La Rue. Essai sur l'économie de la forme urbaine. La Tour d'Aigues, Éditions de l'Aube, $285 \mathrm{p}$.

Monnet J., 2006-2007. La rue et la représentation de la ville : iconographie et lieux communs à Mexico et à Los Angeles, Flux, n 66-67, p. 8-18.

Morelle M., 2007. La Rue des enfants, les enfants des rues : Yaoundé et Antananarivo. Paris, Éditions du CNRS, $282 \mathrm{p}$.

Roulleau-Berger L., Yuhua G., Peilin L., Shiding L. (dir.), 2008. La nouvelle sociologie chinoise. Paris, CNRS Éditions, $500 \mathrm{p}$.

Sanjuan T., 2009. Atlas de Shanghai. Paris, Autrement, 88 p.

Sanjuan T., 2007. Atlas de la Chine : les mutations accélérées. Paris, Autrement, 80 p.

Sanjuan T. (dir.), 2006. Dictionnaire de la Chine contemporaine, Paris, Armand Colin, 304 p. 
Watson J. L., 1997. Golden Arches East: Mc Donald's in East Asia. Stanford, Stanford University Press, $256 \mathrm{p}$.

Wu F., 2005. Villes chinoises en mouvement. Revue Urbanisme, $\mathrm{n}^{\circ} 341, \mathrm{p} .73-75$.

\section{NOTES}

1. Source : le Bureau d'État des Statistiques de la République Populaire de Chine (2008).

2. Gourdon Jean-Loup, La Rue. Essai sur l'économie de la forme urbaine, La Tour d'Aigues, Édition de l'Aube, 2001, p. 14.

3. Watson James L., Golden arches east: Mc Donald's in East Asia, Stanford, Ed. Stanford University Press, 1997, $256 \mathrm{p}$.

4. Cet article s'inscrit dans la continuité du programme de recherche sur « la Rue en Chine : les recompositions de la riveraineté » coordonné par le Professeur Thierry Sanjuan et Antoine Brès, entre le laboratoire PRODIG (UMR 8586), le magistère d'urbanisme et d'aménagement de l'Université Paris I Panthéon-Sorbonne et le Centre d'Architecture et d'Urbanisme de l'Université Tongji (Shanghai).

5. Monnet Jérôme, «La rue et la représentation de la ville : iconographie et lieux communs à Mexico et à Los Angeles ", Flux, nº6-67, 2006-2007, p. 10-11.

6. Bergère Marie-Claire, Histoire de Shanghai, Paris, Fayard, 2002, p. 257.

7. Ibid, p. 71.

8. Ibid, p. 264.

9. Source: China Statistical Yearbook (2000).

10. Goffman Erwing, La Mise en scène de la vie quotidienne, Paris, Éditions de minuit, 2007, 256 p.

11. Brès Antoine, Inscription territoriale des mobilités et riveraineté des voies, thèse d'urbanisme et d'aménagement, Université Paris 1 Panthéon-Sorbonne, octobre 2005.

12. Sanjuan Thierry, Atlas de Shanghai, Paris, Autrement, 2009, p. 40-41.

13. Wu Fulong, «Villes chinoises en mouvement », Revue Urbanisme, $n^{\circ} 341,2005$, p. 73-75.

14. Entretien réalisé dans la rue du Sichuan (Nord), le jeudi 9 juillet 2009.

15. Sanjuan Thierry (dir.), Dictionnaire de la Chine contemporaine, Paris, Armand Colin, 2006, p. 235-236.

16. Sanjuan Thierry, Atlas de Shanghai, Paris, Autrement, 2009, p. 52-53.

17. Entretien réalisé dans une rue adjacente à celle du Sichuan (Nord), le lundi 22 juin 2009.

18. Morelle Marie, La Rue des enfants, les enfants des rues: Yaoundé et Antananarivo, Paris, Éditions du CNRS, 2007, $282 \mathrm{p}$.

19. La classe moyenne chinoise peut se définir par l'ensemble des personnes dont le revenu annuel est compris entre 25000 et 100000 yuans.

20. Enquêtes menées sur un échantillonnage de 140 entretiens entre juin et septembre 2009.

21. Entretien réalisé dans une rue parallèle à celle du Sichuan (Nord), le samedi 18 juillet 2009.

22. Pratique quotidienne : plus de trois fois par semaine.

23. Entretien réalisé dans la rue du Sichuan (Nord), le 29 août 2009.

24. Entretien réalisé dans la rue du Sichuan (Nord), 14 juillet 2009.

25. Damon Julien, Les Indésirables dans les espaces de transport. Les exemples de la RATP et de la SNCF, Recherche effectuée pour le compte de la direction des Grandes Lignes SNCF, sous la direction d'Hervé Laroche, ESCP, 1993, p. 54-63.

26. Entretien réalisé dans la rue du Sichuan (Nord), le 11 juillet 2009.

27. Entretien réalisé dans la rue du Sichuan (Nord), le 5 juillet 2009.

28. Sanjuan Thierry, Atlas de Shanghai, Paris, Autrement, 2009, p. 30-31.

29. Entretien réalisé dans la rue du Sichuan (Nord), le 21 juin 2009. 
30. Sanjuan Thierry, Atlas de Shanghai, Paris, Autrement, 2009, p. 46-47.

31. Entretien réalisé dans un lilong de la rue du Sichuan (Nord), le 22 juillet 2009.

\section{RÉSUMÉS}

Cet article traite des dynamiques urbaines, économiques et sociales à Shanghai, à partir du cas de l'évolution socio-morphologique de trois artères péri-urbaines. Les artères péri-centrales participent pleinement à l'organisation de l'espace shanghaien. Appréhender les évolutions actuelles de la rue chinoise a une double vocation: identifier, d'une part, les dynamiques urbaines des rues; appréhender, d'autre part, les dynamiques sociales. Les mutations des rues permettent d'apprécier concrètement comment les artères péri-urbaines évoluent depuis le début des années 1990 dans le cadre de la redéfinition plus globale de la municipalité, et d'analyser les dynamiques à la fois sociales, politiques et économiques qui sont en jeu.

This article discusses the dynamics of urban, economic and social in Shanghai, from where the socio-morphological evolution of three peri-urban arteries. The peri-central arteries participate fully in the organization of Shanghainese space. Understanding current developments of the Chinese street has a dual mission: to identify, first, the urban dynamics of streets, understanding on the other hand, the social dynamics. Mutations in the streets can actually appreciate how peri-urban arteries evolved since the early 1990's as part of the broader redefinition of the municipality, and to analyze the dynamics of both social, political and economic.

\section{INDEX}

Keywords : gentrification, globalization, homogenization, identity, peri-central arteries, Shanghai, social dynamic, social stratification, street, urban dynamic

Mots-clés : artère péri-centrale, dynamiques sociales, dynamiques urbaines, espace public, gentrification, homogénéisation, identité, mondialisation, rue, Shanghai, stratification sociospatiale

\section{AUTEUR}

\section{HOWARD VAZQUEZ}

Howard Vazquez est doctorant à l'UMR 8586 PRODIG, Université Paris I Panthéon-Sorbonne howard75@hotmail.fr 